\title{
Anti-Epileptic Medication-Induced Asymptomatic Eosinophilic Colitis
}

\author{
Yamam Al-Saadi ${ }^{\mathrm{a}}$, Mohammad Bilal ${ }^{\mathrm{b}, \mathrm{d}}$, David A. Pacheco ${ }^{\mathrm{c}}$, \\ Praveen Guturu ${ }^{\mathrm{b}}$
}

\section{To the Editor}

Eosinophilic colitis (EC) is a rare self-limiting disease characterized by eosinophilic infiltration of the colon. The presentation can vary from abdominal pain, diarrhea and rectal bleeding. We present a unique case of EC secondary to use of anti-epileptic medications.

A 59-year-old male with past history of seizures was referred for a screening colonoscopy. The patient denied any abdominal pain, diarrhea and rectal bleeding. Abdominal examination was normal. Laboratory data were unremarkable except mildly elevated eosinophilic count of $1.5 \times 10^{9} / \mathrm{L}$. Remainder of the complete blood count including white blood cell count, hemoglobin and platelets was normal. Colonoscopy revealed diminutive whitish nodules in the cecum and ascending colon (Fig. 1). Histological examination showed increased eosinophils and abscess formation in the lamina propria suggestive of EC (Fig. 2). Giemsa staining did not reveal any parasites and no lymphoid hyperplasia was seen. Our patient was using levetiracetam, valproic acid and oxcarbazepine for his seizures, but had been previously on carbamazepine which was discontinued 4 years ago. The patient does not use any other medications or over the counter supplements. Patient continues to be asymptomatic and no treatment has been initiated.

EC can present with a variety of symptoms. About onethird of the patients remain asymptomatic as was the case in our patient. Previously carbamazepine has been associated with EC along with hypersensitivity syndrome [1]. Our patient had not been using carbamazepine for the past 4 years. Although all the medications being used by our patient are known to cause peripheral eosinophilia, these anti-epileptic medications have not previously been associated with EC. Our report shows that an isolated EC can occur in the setting of anti-epileptic medication usage. Our patient did not have any other manifestations of hypersensitivity syndrome. It is important for gastroenterologists to be aware of this subtle appearance of EC which can mimic lymphoid hyperplasia, so biopsies can be obtained in the appropriate setting.

\section{Conflict of Interest}

The authors of the above manuscript have no conflicts of interest.

\section{References}

1. Atkinson RJ, Dennis G, Cross SS, McAlindon ME, Sharrack B, Sanders DS. Eosinophilic colitis complicating anti-epileptic hypersensitivity syndrome: an indication for colonoscopy? Gastrointest Endosc. 2004;60(6):10341036.

aDepartment of Internal Medicine, University of Texas Medical Branch, Galveston, TX, USA

${ }^{b}$ Department of Gastroenterology \& Hepatology, University of Texas Medical Branch, Galveston, TX, USA

'Department of Pathology, University of Texas Medical Branch, Galveston, TX, USA

${ }^{\mathrm{d} C}$ Corresponding Author: Mohammad Bilal, 7400 Jones Drive, Apt 724, Galveston, TX 77551, USA. Email:mobilal@utmb.edu 


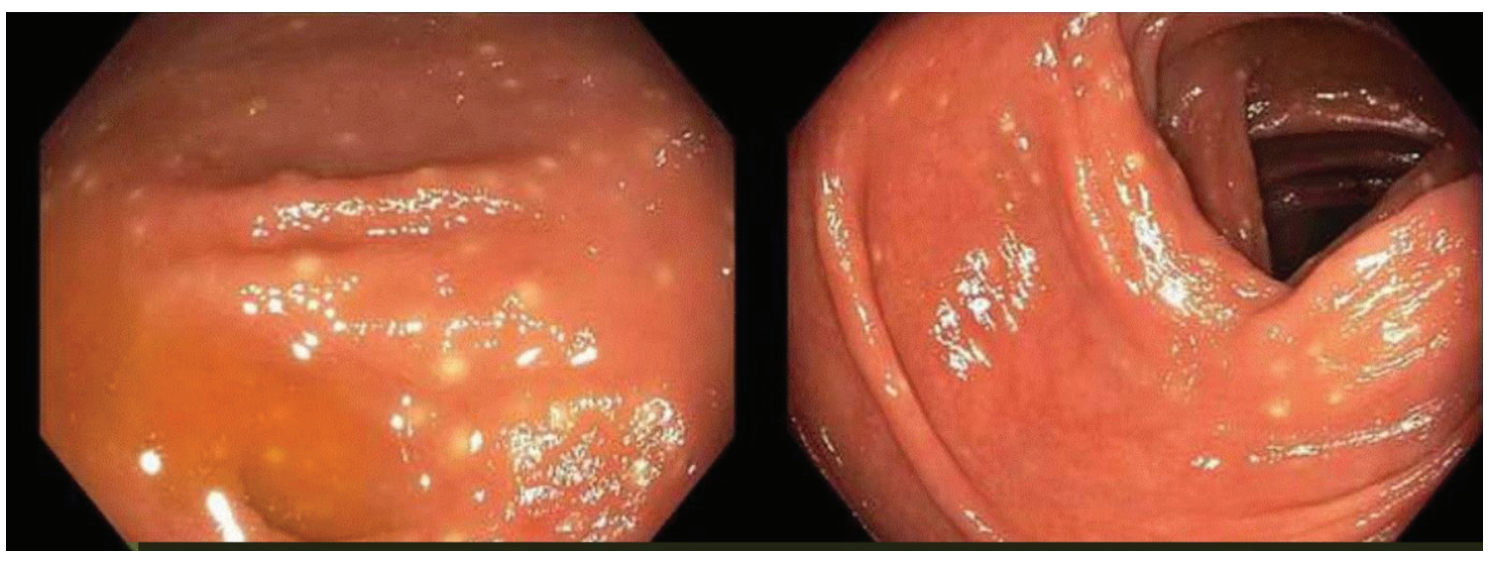

Figure 1. Colonoscopy showing diminutive whitish nodules in the cecum and ascending colon.

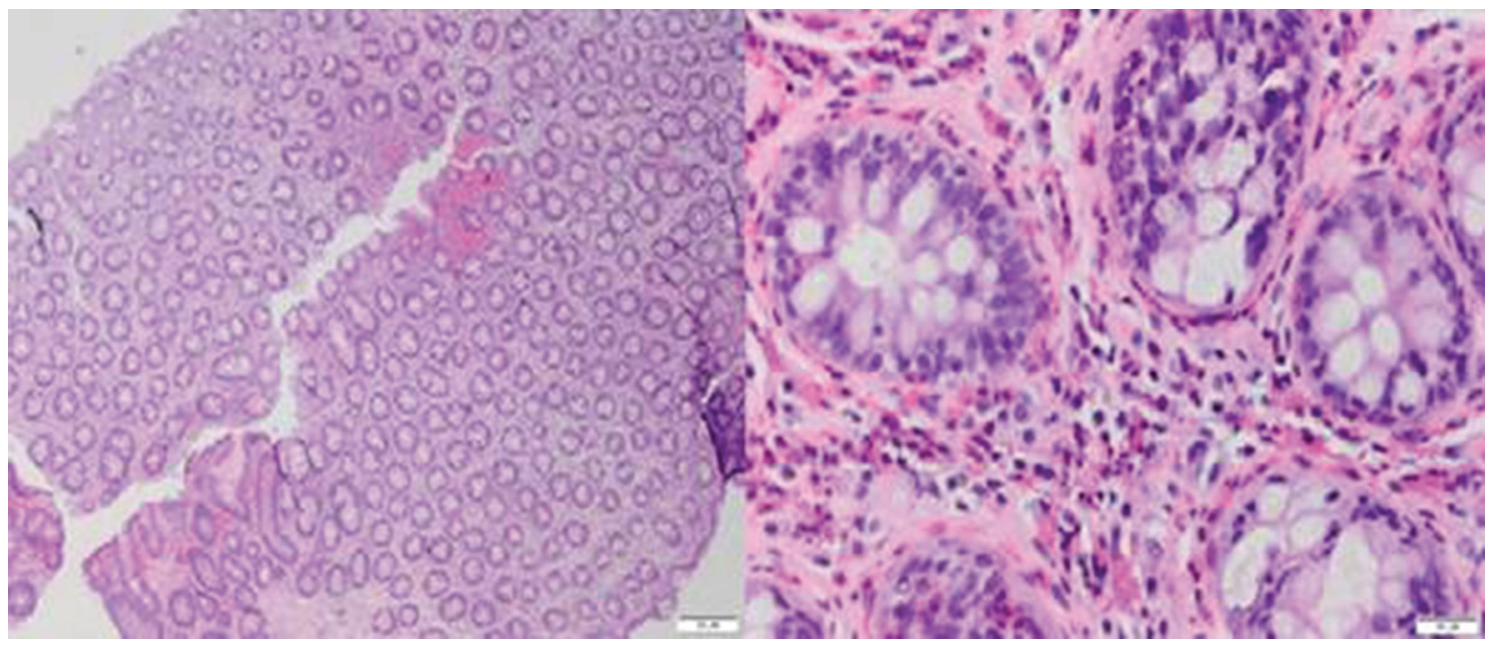

Figure 2. (Left) Low magnification $(\times 2)$. H\&E stain. Specimen showing increased eosinophils in the lamina propria. (Right) High magnification $(\times 20)$. H\&E stain. Extensive eosinophilic infiltration of lamina propria with eosinophilic cryptitis. 